\title{
Sites of Alkylation of Human Keap1 by Natural Chemoprevention Agents
}

\author{
Yan Luo, Aimee L. Eggler, Dongting Liu, Guowen Liu, \\ Andrew D. Mesecar, and Richard B. van Breemen \\ Department of Medicinal Chemistry and Pharmacognosy, University of Illinois College of Pharmacy, \\ Chicago, Illinois, USA
}

Under basal conditions, the interaction of the cytosolic protein Keap1 (Kelch-like ECHassociated protein 1) with the transcription factor nuclear factor- $\mathrm{E}_{2}$-related factor 2 (Nrf2) results in a low level of expression of cytoprotective genes whose promoter region contains the antioxidant response element (ARE). Alkylation of one or more of the 27 cysteine sulfhydryl groups of human Keap1 is proposed to lead to Nrf2 nuclear accumulation, to upregulation of cytoprotective gene expression by the ARE, and to prevention of degenerative diseases, such as cancer. Therefore, identification of the most reactive of these cysteine residues toward specific electrophiles should help clarify this mechanism of cancer prevention, also known as chemoprevention. To address this issue, preliminary analyses of tryptic digests of Keap1 alkylated by the model electrophile 1-biotinamido-4-(4'-[maleimidoethyl-cyclohexane]carboxamido) butane were carried out using liquid chromatographic-tandem mass spectrometry (LC-MS/MS) with a cylindrical ion trap mass spectrometer and also using LC-MS/MS with a hybrid linear ion trap FT ICR mass spectrometer. Because the FT ICR instrument provided more complete peptide sequencing coverage and enabled the identification of more alkylated cysteine residues, only this instrument was used in subsequent studies of Keap1 alkylation by three electrophilic natural products that can upregulate the ARE, xanthohumol, isoliquiritigenin, and 10-shogaol. Among the various cysteine residues of Keap1, C151 was most reactive toward these three electrophiles. These in vitro results agree with evidence from in vivo experiments, and indicate that C151 is the most important site of alkylation on Keap1 by chemoprevention agents that function by activating the ARE through Nrf2. (J Am Soc Mass Spectrom 2007, 18, 2226-2232) (C) 2007 American Society for Mass Spectrometry

$\mathrm{K}$ elch-like ECH-associated protein 1 (Keap1) is a cytoplasmic protein that sequesters the transcription factor nuclear factor- $\mathrm{E}_{2}$-related factor 2 (Nrf2) in the cytosol under basal conditions [1]. Nrf2 is a member of the NF-E2 family of nuclear basic leucine zipper (bZIP) transcription factors. By binding to the 5 '-upstream regulatory antioxidant response element (ARE) regions of cytoprotective genes, Nrf2 upregulates their transcription $[2,3]$, thus protecting cells against damage by reactive oxygen intermediates or other electrophilic species [4]. Keap1 functions as a bridge between Nrf2 and the Cullin3-based E3-ligase ubiquitination complex, promoting ubiquitination and subsequent proteasomal degradation of Nrf2 [5-7], thus preventing nuclear accumulation of Nrf2. Without the intervention of Keap1, levels of Nrf2 are elevated in the nucleus causing upregulation of the ARE [8]. Alkylation of one or more of the 27 cysteine residues of Keap 1 by reactive oxygen species and xenobiotic electrophiles, including chemopreventive agents, appears to be an

Address reprint request to Professor Richard B. van Breemen, University of Illinois College of Pharmacy, Department of Medicinal Chemistry and Pharmacognosy, 833 S. Wood Street, Chicago, IL 60612, USA. E-mail: breemen@uic.edu important signaling mechanism for the regulation of the ARE through Nrf2 [3].

Keap1 has five distinct domains: the $\mathrm{N}$-terminal domain (amino acids 1-60); the BTB (Bric-a-brac, Tramtrack, Broad-complex) domain (amino acids 61178); a central linker domain (amino acids 179-321); the Kelch repeat domain (amino acids 322-608); and a C-terminal domain (amino acids 609-625). The BTB domain mediates the dimerization of Keap1 [9] and also binds the adaptor protein in Cul3-dependent ubiquitination systems [10]. The Kelch repeat domain binds to the Nrf2 directly [1]. Because Keap1 signaling is probably mediated by alkylation of one or more of its cysteine sulfhydryl groups [3,11, 12], identification of the cysteine residues that are most reactive toward specific electrophiles should help clarify this mechanism of action of Keap1.

However, most of the work to date to identify reactive Keap1 cysteine residues has been carried out with model alkylating agents such as dexamethasone 21-mesylate [2], iodoacetyl-N-biotinyl hexylene diamine (BIA), and 1-biotinamido-4-(4'-[maleimidoethylcyclohexane]-carboxamido) butane (BMCC) [13] instead of biologically relevant ARE inducers that show promise as chemopreventive agents. Unlike ARE induc- 
<smiles>O=C(/C=C/c1ccccc1)c1ccc(O)cc1O</smiles>

Isoliquiritigenin CD $1.8 \pm 0.44 \mu \mathrm{M}$

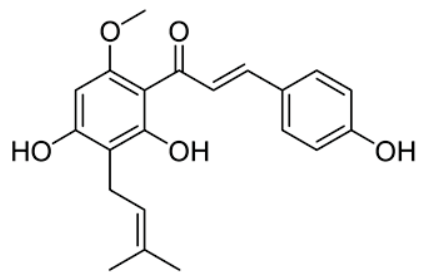

Xanthohumol

CD $1.7 \pm 0.7 \mu \mathrm{M}$
$\mathrm{C}$<smiles>CCCCCCCCCC(=O)C=CCc1ccc(O)c(OC)c1</smiles>

10-Shogaol

CD $42.4 \pm 0.9 \mu \mathrm{M}$

Scheme 1. Structures of the natural product chemoprevention agents (A) isoliquiritigenin [22]; (B) xanthohumol [28]; and (C) 10-shogaol [29]. The CD values represent the concentration of each compound required to double the intracellular quinone reductase activity in Hepa 1c1c7 murine hepatoma cells [22] and indicate the relative activities of these compounds as chemopreventive reagents.

ers that are Michael addition acceptors, alkylation by dexamethasone 21-mesylate and BIA is irreversible, which means that sample preparation would not lead to dissociation of such adducts. Because BMCC and BIA are biotinylated compounds, avidin-biotin affinity chromatography may be used to facilitate the isolation and identification of peptides alkylated by these electrophiles. However, few if any chemoprevention agents contain biotin tags to facilitate analysis. Therefore, a general analytical method for the characterization of alkylated Keap1 must minimize decomposition of adducts while facilitating the identification of alkylation sites without the benefit of affinity chromatography.

In this investigation, a method based on liquid chromatographic-tandem mass spectrometry (LC-MS/ MS) was developed that facilitated the detection of cysteine residues in human Keap1 modified by chemopreventive agents. Because it was anticipated that partial purification of the alkylated peptides from Keap1 might be necessary before LC-MS/MS analysis to detect all alkylated cysteine residues, preliminary analyses were carried out of Keap1 that had been alkylated by the model biotinylated electrophile BMCC using a cylindrical ion trap mass spectrometer (LCQ) or a linear ion trap-FI ICR mass spectrometer (LTQ-FT ICR). More BMCC alkylated cysteine residues in human Keap1 could be identified using the LTQ-FT ICR mass spectrometer without affinity isolation than when using either instrument with affinity purification. Therefore, this method was applied to the analysis of the reaction products between human Keap1 and each of three natural products containing Michael acceptors, xanthohumol, isoliquiritigenin, and 10-shogaol (see structures in Scheme 1).

Isolated from the hop plant, Humulus lupulus L. (Cannabaceae), xanthohumol has been reported to be a cancer chemopreventive agent with activity both in vitro and in vivo [14-17]. Isoliquiritigenin is a constituent of the tonka bean Dipteryx odorata (Aubl.) Willd, and is also present in licorice (Glycyrrhiza uralensis) [18]. Isoliquiritigenin has been reported to have chemopre- vention activity in both in vitro and several animal models [19-22]. 10-Shogaol is a constituent of the rhizome of Zingiber officinale (ginger) [23]. Although not attributed specifically to 10-shogaol, ginger has been reported to have antioxidant and anticarcinogenic properties by several groups [24-27]. All three of these natural products contain electrophilic unsaturated ketones (Scheme 1). As an indication of their chemopreventive activity through the induction of ARE, the induction of the phase 2 enzyme quinone reductase in Hepa 1c1c7 murine hepatoma cells by xanthohumol, isoliquiritigenin, and 10-shogaol has been determined to be $1.7 \pm 0.7,1.8 \pm 0.44$, and $42.4 \pm 0.9 \mu \mathrm{M}$, respectively $[22,28,29]$. In this investigation, the relative reactivities of each of these compounds toward specific cysteine residues of human Keap1 were determined using LCMS/MS, and the most reactive amino acid residue was identified as C151 for all three compounds.

\section{Experimental}

\section{Materials and Reagents}

Recombinant human Keap1 protein containing a histidine tag was expressed in and purified from Escherichia coli. The details of the cloning, expression, and purification of human Keap1 are described elsewhere [30]. BMCC was purchased from Pierce (Rockford, IL), and trypsin was purchased from Promega (Madison, WI). An ICAT $^{\mathrm{TM}}$ kit for protein labeling was purchased from Applied Biosystems (Foster City, CA). Deionized water was prepared using a Milli-Q purification system (Millipore, Bedford, MA). Acetonitrile (Optima) was purchased from Fisher Scientific (Hanover Park, IL). Isoliquiritigenin, dimethyl sulfoxide, tris(carboxylethyl)phosphine, D,L-dithiothreitol (DTT), and iodoacetamide were purchased from Sigma-Aldrich (St. Louis, $\mathrm{MO}$, and Milwaukee, WI). 10-Shogaol was isolated and provided by Dr. Yi Tao of the University of Illinois College of Pharmacy, and xanthohumol was isolated and pro- 
vided by Dr. Luke R. Chadwick and Dr. Guido F. Pauli of the same institution.

\section{Sample Preparation}

Alkylation of Keap1 by isoliquiritigenin, xanthohumol, 10-shogaol, or BMCC (as a positive control) was carried out at different ratios as follows (using BMCC as an example). A stock solution of BMCC was prepared at a concentration of $10 \mu \mathrm{M}$ in dimethyl sulfoxide. Human Keap1 $(10 \mu \mathrm{M})$ was incubated with BMCC at molar ratios from 1:0.025 to 1:2 ([Keap1]/[BMCC]) at room temperature for $2 \mathrm{~h}$ in $100 \mu \mathrm{L} 25 \mathrm{mM}$ Tris buffer $(\mathrm{pH}$ 8.0). Next, DTT was added to this reaction mixture at a concentration of $1 \mathrm{mM}$. After incubation for $30 \mathrm{~min}$, excess iodoacetamide was added to block the remaining free cysteines in Keap1. Subsequently, 5 mM DTT was added to quench unreacted iodoacetamide. Finally, 0.5 $\mu \mathrm{g}$ trypsin was added to each Keap1 solution followed by incubation at $37^{\circ} \mathrm{C}$ for $3 \mathrm{~h}$ (BMCC) or $1.5 \mathrm{~h}$ (all other electrophiles). The tryptic peptides were analyzed using LC-MS/MS. Alternatively, BMCC modified peptides were purified from the tryptic digest of Keap1 using an avidin affinity column as described by the manufacturer of the ICAT kit before analysis by LC-MS/MS.

\section{LC-MS/MS Analyses Using a Cylindrical Ion Trap Mass Spectrometer}

Peptides were analyzed initially using a Finnigan $\mathrm{LCQ}^{\text {Deca }}$ (Thermo, San Jose, CA) ion trap mass spectrometer equipped with a Surveyor HPLC system, and a Vydac (Hesperia, CA, USA) 218MS LC-MS C 18 reversed-phase HPLC column $(5 \mu \mathrm{M}, 2.1 \times 150 \mathrm{~mm}, 300 \AA)$ and a $218 \mathrm{TP}$ $(5 \mu \mathrm{M}, 2.1 \mathrm{~mm}$ ) guard column. The solvent system consisted of a linear gradient from 5 to $45 \%$ solvent B over 60 min and then $45-80 \%$ solvent B over 15 min (solvent A: 95:4.9:0.1; and solvent B: 4.9:95:0.1, water/acetonitrile/ formic acid, vol/vol/v) at a flow rate of $200 \mu \mathrm{L} / \mathrm{min}$. The LCQ mass spectrometer was operated in a data-dependent MS/MS mode in which the most abundant peptide ion in each mass spectrum was selected for collisioninduced dissociation (CID) using a normalized collision energy of $35 \%$. The LC-MS/MS data were processed using Thermo BioWorks ${ }^{\mathrm{TM}} 3.1$ and TurboSEQUEST. The sites of Keap1 alkylation were identified based on both automated data processing and manual inspection of the tandem mass spectra.

\section{LC-MS/MS Analyses Using a Linear Ion Trap-FT ICR Hybrid Mass Spectrometer}

For confirmation of the data obtained using the cylindrical ion trap, LC-MS/MS analysis of the alkylated Keap1 peptides was also carried out using a high-resolution Finnigan LTQ-FT ICR mass spectrometer equipped with a Dionex (Auburn, CA) microcapillary HPLC system. The same HPLC separation conditions were used as described earlier except that a 35-min linear gradient was used from the $5-45 \%$ solvent B. The LTQ-FT ICR mass spectrometer was operated in a data-dependent MS/MS mode in which the five most abundant peptide ions in each mass spectrum were selected for CID using a normalized collision energy of $35 \%$.

\section{Results and Discussion}

\section{Cysteines in Keap1 Modified by BMCC}

Because Keap1 has a molecular mass exceeding 70,000 $\mathrm{Da}$, tryptic digestion formed in excess of 100 peptides (calculated using one missing cleavage site). To detect all the modified peptides, it was anticipated that partial purification of the modified peptides would be necessary such as ion-exchange fractionation or affinity extraction before LC-MS/MS analysis. To determine whether an affinity purification step was necessary, a preliminary study was carried out using Keap 1 that had been alkylated by the model electrophilic biotinylated molecule BMCC using a cylindrical ion trap mass spectrometer (LCQ). As shown in Table 1, avidin purification usually increased the number of modified peptides detected, though not to a great extent.

Upon acquisition of a linear ion trap FT ICR mass spectrometer (LTQ-FT ICR MS), the study was updated using this new instrument for LC-MS/MS analysis. Due to its faster scan speed and greater sensitivity, more biotinylated peptides were identified using the LTQ-FT ICR than the LCQ mass spectrometer, especially when the ratio of BMCC to protein was low (see Table 1). Routinely, $90 \%$ of the protein sequence was covered by MS/MS analysis using the LTQ-FT ICR mass spectrometer (compared to only 75 to $80 \%$ of sequence coverage using the LCQ mass spectrometer), and all the cysteinecontaining tryptic peptides were detected. Comparing the alkylation sites found by using the LCQ and LTQ-FT mass spectrometers at the same molar ratio of BMCC and Keap1, more modified sites were detected using the LTQ-FT mass spectrometer (Table 1). Interestingly, at low ratios, avidin purification actually reduced the number of detected peptides. This might be due to the high affinity of the biotin-avidin complex $\left(K_{\mathrm{d}} \approx\right.$ $10^{-15} \mathrm{M}^{-1}$ ) [31], which perhaps retained some of the biotinylated peptides on the avidin column and caused some BMCC modified peptides to be lost during the affinity purification. A more probable possible cause of the loss of BMCC modified peptides is dissociation of the BMCC-cysteine adducts during affinity purification. Given that BMCC is a Michael addition acceptor, it can modify cysteine residues reversibly. Therefore, during avidin-biotin affinity purification, some of the adducts probably dissociated before they could be detected.

FT ICR mass spectrometry greatly facilitated the identification of alkylated cysteine residues in human Keap1, in particular, those that could not be purified by affinity means. Therefore, this FT ICR mass spectrometer-based method was used without affinity column purification in 
Table 1. Alkylation of cysteine residues in human Kelch-like ECH-associated protein 1 (Keap1) by 1-biotinamido-4-(4'[maleimidoethyl-cyclohexane]-carboxamido) butane (BMCC) as a function of BMCC concentration ${ }^{\text {a }}$

\begin{tabular}{|c|c|c|c|}
\hline$[\mathrm{BMCC}] /[$ Keap1] & $\begin{array}{l}\text { Avidin } \\
\text { purification }\end{array}$ & $\begin{array}{l}\text { Modified cysteines detected using the cylindrical } \\
\text { ion trap MS }\end{array}$ & $\begin{array}{c}\text { Modified cysteines detected using the linear } \\
\text { ion trap-FT ICR MS }\end{array}$ \\
\hline 0.025 & No & None ${ }^{b}$ & $\mathrm{C} 151, \mathrm{C} 434^{\mathrm{C}}$ \\
\hline 0.025 & Yes & None & None \\
\hline 0.05 & No & None & C151, C434 \\
\hline 0.05 & Yes & None & None \\
\hline 0.1 & No & None & C151, C434, C288, C319, C613 \\
\hline 0.1 & Yes & None & None \\
\hline 0.5 & No & C151 & $\begin{array}{l}\text { C151, C434, C288, C319, C613, C226, C257, } \\
\text { C297 }\end{array}$ \\
\hline 0.5 & Yes & None & C434, C288, С319, C297. \\
\hline 1 & No & C151, C319, C273, C257, C297 & $\begin{array}{l}\text { C151, C434, C288, C319, C613, C226, C257, } \\
\text { C297, C77, C273 }\end{array}$ \\
\hline 1 & Yes & C151, C319, C273, C257, C297, C613, C226 & $\begin{array}{l}\text { C151, C434, C288, C319, C613, C226, C257, } \\
\text { C297, C77, C273 }\end{array}$ \\
\hline 2 & No & C151, C319, C273, C257, C297, C613, C226, C434 & $\begin{array}{l}\text { C151, C434, C288, C319, C613, C226, C257, } \\
\text { C297, C77, C273, C23, C38 }\end{array}$ \\
\hline 2 & Yes & $\begin{array}{l}\text { C151, C319, C273, C257, C297, C613, C226, C434, } \\
\text { C622, C288, C241, C23 }\end{array}$ & $\begin{array}{l}\text { C151, C434, C288, C319, C613, C226, C257, } \\
\text { C297, C77, C273, C23, C38 }\end{array}$ \\
\hline
\end{tabular}

aBMCC was incubated with Keap1 at different molar ratios, and at each molar ratio, the tryptic peptides of Keap1 were analyzed using two instruments: a cylindrical ion trap mass spectrometer and a linear ion trap-FT ICR mass spectrometer.

b"None" indicates that no peptides containing BMCC modified cysteine residues were detected.

"Peptides detected containing BMCC modified cysteine. The order in which these modified cysteines are listed corresponds to their relative abundances.

all subsequent experiments for the analysis of the reactivities of Keap1 cysteines toward ARE inducers.

\section{Modification Sites of Human Keap1 by Electrophiles}

Because FT ICR LC-MS/MS facilitated the identification of sites of cysteine alkylation by electrophiles in human Keap1 without the requirement of affinity column enrichment of alkylated peptides, this instrument was used for the detection of the alkylation sites in Keap1 after reaction with electrophiles lacking affinity tags such as biotin. Specifically, this method was used to determine the modification pattern of Keap1 after alkylation by ARE inducers containing a Michael addition moiety. Three different electrophilic natural products-isoliquiritigenin, xanthohumol, and 10-shogaol (Scheme 1)-were incubated with Keap1. Incubations with a range of concentrations of these electrophiles were used to determine through a titration-like process which Keap1 cysteine residues became alkylated first. Trypsin digestion followed by peptide mapping and sequencing of the alkylated Keap1 protein were carried out to determine the sites of alkylation. As expected, only cysteine residues were alkylated, and these residues are shown in Table 2. The three most readily modified cysteines of human Keap1 by xanthohumol were identified as C151, C319, and C613. The two most readily modified cysteines of human Keap1 by isoliquiritigenin were identified as C151 and C226. The three most readily modified cysteines of human Keap1 by 10-shogaol were C151, C257, and C368. The tandem mass spectra of the peptide ions containing C151 resi- dues that had been alkylated by xanthohumol, isoliquiritigenin, or 10-shogaol are shown in Figure 1.

Before the discovery of Keap1 protein, Talalay et al. [32] had proposed that there must be a protein with highly reactive cysteine thiols that acts as a sensor for phase 2 inducers. Since the isolation of Keap1 from a yeast system using a Gal4-Neh2 fusion protein [33], great interest has focused on the reactivity of Keap1 cysteine residues. Dinkova-Kostova et al. [2] studied the reaction of murine Keap1 with dexamethasone mesylate using mass spectrometry and suggested that C257, C273, C288, and C297 were the most reactive sites among the 25 murine Keap 1 cysteine residues. In their studies of murine Keap1, Dinkova-Kostova et al. [2] used MALDI-TOF to identify modified peptides in a tryptic digest, but LC-MS/MS was used to confirm the sequence and to determine the site of modification in only two of the four modified peptides. Our previous study [30] in which human Keap1 modifications were mapped indicated that C151, C288, and C297 are the most reactive residues toward iodoacetyl-N-biotinyl hexylene diamine. A similar study was reported by Hong et al. [13], but their data suggested that C169, C241, and C288 but not C151 were reactive toward human Keap1. Subsequently, we found that a limitation of the sample preparation procedure used by Hong et al. [13] had resulted in their inability to detect the modified C151 peptide [36]. Specifically, Hong et al. [13] used an ultracentrifugation device for buffer exchange, which resulted in the binding and loss of $>99 \%$ of the protein due to adsorption and possibly changed the conformation of Keap1 so that a different modification pattern was detected [36]. 
Table 2. Cysteine residues in Keap1 modified by chemopreventive agents as indicated by LC-MS/MS analysis

\begin{tabular}{|c|c|c|c|c|c|c|c|c|c|c|}
\hline \multirow[b]{2}{*}{ Domain } & \multirow[b]{2}{*}{ Cysteine } & \multicolumn{3}{|c|}{ [Xanthohumol]/[Keap1] } & \multicolumn{3}{|c|}{ [Isoliquiritigenin]/[Keap1] } & \multicolumn{3}{|c|}{ [10-Shogaol]/[Keap1] } \\
\hline & & 5 & 10 & 20 & 2 & 5 & 10 & 2 & 5 & 10 \\
\hline \multirow[t]{4}{*}{ N-terminal } & C13 & & & & & & & & & 1,2 \\
\hline & C14 & & & & & & & & & 1,2 \\
\hline & $\mathrm{C} 23$ & & & & & 1,2 & $1,2,3$ & & & 2,3 \\
\hline & C38 & & & 1,2 & & & 1 & & & 2,3 \\
\hline \multirow[t]{3}{*}{ BTB } & $\mathrm{C} 77$ & & 3 & 3 & & $1,2,3$ & $1,2,3$ & & & \\
\hline & C151 & $1,2,3$ & $1,2,3$ & $1,2,3$ & $1,2,3$ & $1,2,3$ & $1,2,3$ & $1,2,3$ & $1,2,3$ & $1,2,3$ \\
\hline & C171 & & & & & & & & & \\
\hline \multirow[t]{9}{*}{ Central linker } & C196 & & & & & 1,2 & $1,2,3$ & & & \\
\hline & $\mathrm{C} 226$ & & & & 1,2 & $1,2,3$ & $1,2,3$ & & & $1,2,3$ \\
\hline & $\mathrm{C} 241$ & & & & & & & & $1,2,3$ & $1,2,3$ \\
\hline & C249 & & & & & & 1 & & $1,2,3$ & $1,2,3$ \\
\hline & $\mathrm{C} 257$ & & & & & & & $1,2,3$ & $1,2,3$ & $1,2,3$ \\
\hline & $\mathrm{C} 273$ & & & & & & & & & 1,3 \\
\hline & $\mathrm{C} 288$ & & & & & & & & & \\
\hline & C297 & & & & & & & & & \\
\hline & C319 & $1,2,3$ & $1,2,3$ & 1,2 & 3 & $1,2,3$ & $1,2,3$ & & $1,2,3$ & $1,2,3$ \\
\hline \multirow[t]{8}{*}{ Kelch } & C368 & & & & & & 1,3 & $1,2,3$ & $1,2,3$ & $1,2,3$ \\
\hline & C395 & & & & & 1,2 & $1,2,3$ & & & \\
\hline & $\mathrm{C} 406$ & & & & & & & & & \\
\hline & $\mathrm{C} 434$ & & 1,2 & $1,2,3$ & & & 1,3 & & $1,2,3$ & $1,2,3$ \\
\hline & C489 & & & $1,2,3$ & & 2 & $1,2,3$ & & & $1,2,3$ \\
\hline & C513 & & & 3 & & 1,2 & $1,2,3$ & & & \\
\hline & C518 & & & & & 1,2 & $1,2,3$ & & & \\
\hline & C583 & & & & 2 & $1,2,3$ & $1,2,3$ & & & \\
\hline \multirow[t]{3}{*}{ C-terminal } & $\mathrm{C} 613$ & $1,2,3$ & $1,2,3$ & $1,2,3$ & & 3 & $1,2,3$ & & $1,2,3$ & $1,2,3$ \\
\hline & C622 & & & & & & & & $1,2,3$ & $1,2,3$ \\
\hline & C624 & & & & & & & & $1,2,3$ & $1,2,3$ \\
\hline
\end{tabular}

These data represent the results of LC-MS/MS analyses of three identical experiments. The numbers indicate the experiment numbers (1, 2, and 3 ) in which specific modified cysteines were detected.

We have developed an LC-MS/MS method to characterize the reactivity of human Keap1 toward Michael addition reaction acceptors from natural products that upregulate the ARE. Furthermore, this approach facilitated the identification of the most reactive amino acid residues in Keap1. Although the Keap1 alkylation pattern was unique for each electrophile, only cysteine residues were alkylated, and C151 was always detected among the top three most reactive cysteines. At a ratio of electrophile to Keap1 of only 2:1, which was the smallest ratio tested, 10-shogal and isoliquiritigen but not xanthohumol alkylated C151 to a detectable level. As the ratio of electrophile to Keap1 in the incubation was increased, cysteine modifications by isoliquiritigen and 10-shogal were detected on all five protein domains. Xanthohumol was the least reactive of the three electrophiles; no modification sites were detected at a ratio of 2 to 1, but at a ratio 5 to 1, C151 (located in BTB domain), and C319 (located in central linker domain) and C613 (located in Kelch repeat domain) were modified.

The differences among adduct patterns of these three electrophiles may be due to their unique structures and reactivities. Regardless, these results indicate C151 is extremely reactive toward these three chemoprevention agents. Located in the BTB domain of Keap1, sitedirected mutagenesis studies have indicated that C151 is essential for inhibition of Keap1-dependant ubiquitination and degradation of $\mathrm{Nrf2}$ in response to all tested agents, including tBHQ and sulforaphane [3], NEPP11, an endogenous neurite outgrowth-promoting prostaglandin [34], and ebselen, a seleno-organic drug [35]. Taken together, C151 plays a critical role in the Keap1Nrf2 signaling system, and our results support the view that C151 is a target for Nrf2 activation by Michael addition reaction acceptors that function as ARE inducers.

\section{Conclusions}

The use of FT ICR LC-MS/MS facilitated the identification of sites of cysteine alkylation by electrophiles in human Keap1 digested with trypsin without the need for affinity column enrichment of alkylated peptides. The reaction of Keap1 with the Michael addition reaction acceptors xanthohumol, isoliquiritigenin, and 10shogaol, which are natural products that upregulate the ARE, was investigated using this method. All three compounds alkylated Keap1 at specific cysteine residues, and the most reactive site of Keap1 was C151. This information suggests that natural product chemoprevention agents that are Michael addition reaction acceptors upregulate the ARE as a result of alkylation of Keap1 at C151. 


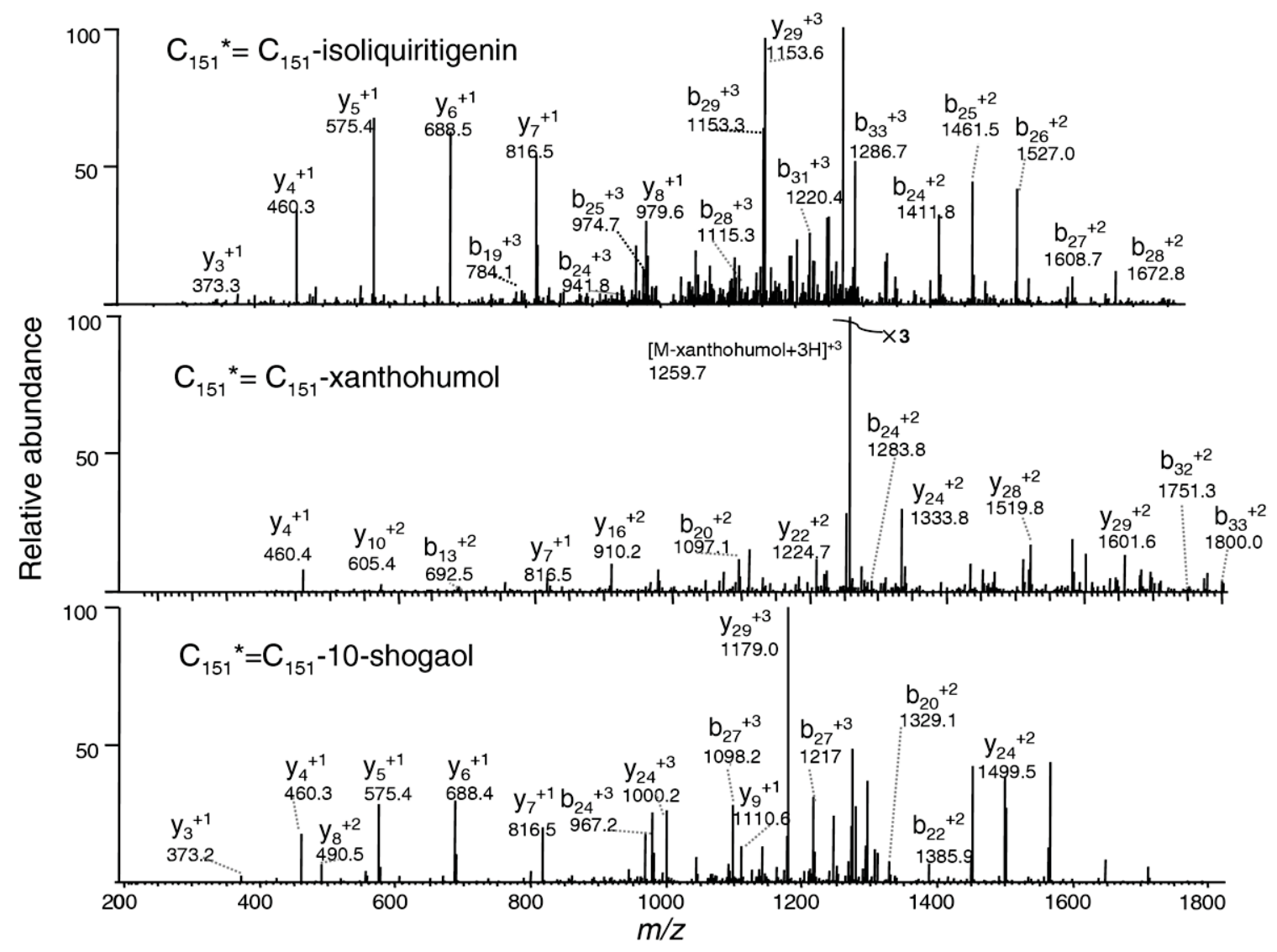

Figure 1. MS/MS spectra of alkylated Keap1 C151 peptides of the sequence LIEFAYTASISMGEKC ${ }_{151}{ }^{*}$ VLHVMNGAVMYQIDSVVR. Keap1 was alkylated by the electrophilic natural products xanthohumol, isoliquiritigenin, or 10-shogaol and then digested with trypsin. $\mathrm{Cys}_{151}{ }^{*}$ represents the site of modification by each electrophilic natural product. All of the peptide fragment ions $y_{19}$ to $y_{33}$ and $b_{16}$ to $b_{33}$ contained the modification.

\section{Acknowledgments}

This work was support by Grant P01 CA-48112 from the National Cancer Institute and Grant P50 AT-00155 provided to the UIC/ $\mathrm{NIH}$ Center for Botanical Dietary Supplements Research by the Office of Dietary Supplements, the Office for Research on Women's Health, and the National Center for Complementary and Alternative Medicine. The authors thank the Chicago Biomedical Consortium for access to the LTQ-FT ICR mass spectrometer, which was purchased with a grant from The Searle Funds at The Chicago Community Trust.

\section{References}

1. Itoh, K.; Wakabayashi, N.; Katoh, Y.; Ishii, T.; Igarashi, K.; Engel, J. D.; Yamamoto, M. Keap1 Represses Nuclear Activation of Antioxidant Responsive Elements by Nrf2 through Binding to the Amino-Terminal Neh2 Domain. Genes Dev. 1999, 1, 76-86.

2. Dinkova-Kostova, A. T.; Holtzclaw, W. D.; Cole, R. N.; Itoh, K.; Wakabayashi, N.; Katoh, Y.; Yamamoto, M.; Talalay, P. Direct Evidence That Sulfhydryl Groups of Keap1 Are the Sensors Regulating Induction of Phase 2 Enzymes that Protect Against Carcinogens and Oxidants. Proc. Natl. Acad. Sci. U. S. A. 2002, 18, 11908-11913.

3. Zhang, D. D.; Hannink, M. Distinct Cysteine Residues in Keap1 are Required for Keap1-Dependent Ubiquitination of Nrf2 and for Stabilization of Nrf2 by Chemopreventive Agents and Oxidative Stress. Mol. Cell. Biol. 2003, 22, 8137-8151.

4. Talalay, P.; Fahey, J. W.; Holtzclaw, W. D.; Prestera, T.; Zhang, Y. Chemoprotection Against Cancer by Phase 2 Enzyme Induction. Toxicol. Lett. 1995, 75, 173-179.

5. Cullinan, S. B.; Gordan, J. D.; Jin, J.; Harper, J. W.; Diehl, J. A. The Keap1-BTB Protein Is an Adaptor that Bridges Nrf2 to a Cul3-Based E3
Ligase: Oxidative Stress Sensing by a Cul3-Keap1 Ligase. Mol. Cell. Biol. 2004, 19, 8477-8486.

6. Furukawa, M.; Xiong, Y. BTB Protein Keap1 Targets Antioxidant Transcription Factor Nrf2 for Ubiquitination by the Cullin 3-Roc1 Ligase. Mol. Cell. Biol. 2005, 1, 162-171.

7. Zhang, D. D.; Lo, S. C.; Cross, J. V.; Templeton, D. J.; Hannink, M. Keap1 is a Redox-Regulated Substrate Adaptor Protein for a Cul3-Dependent Ubiquitin Ligase Complex. Mol. Cell. Biol. 2004, 24, 10941-10953.

8. Itoh, K.; Wakabayashi, N.; Katoh, Y.; Ishii, T.; O'Connor, T.; Yamamoto, M. Keap1 Regulates both Cytoplasmic-Nuclear Shuttling and Degradation of Nrf2 in Response to Electrophiles. Genes Cells 2003, 4, 379-391.

9. Zipper, L. M.; Mulcahy, R. T. The Keap1 BTB/POZ Dimerization Function is Required to Sequester Nrf2 in Cytoplasm. J. Biol. Chem. 2002, $39,36544-36552$.

10. Dinkova-Kostova, A. T.; Holtzclaw, W. D.; Kensler, T. W. The Role of Keap1 in Cellular Protective Responses. Chem. Res. Toxicol. 2005, 12, 1779-1791.

11. Wakabayashi, N.; Dinkova-Kostova, A. T.; Holtzclaw, W. D.; Kang, M. I.; Kobayashi, A.; Yamamoto, M.; Kensler, T. W.; Talalay, P. Protection Against Electrophile and Oxidant Stress by Induction of the Phase 2 Response: Fate of Cysteines of the Keap1 Sensor Modified by Inducers. Proc. Natl. Acad. Sci. U. S. A. 2004, 7, 2040-2045.

12. Hong, F.; Freeman, M. L.; Liebler, D. C. Identification of Sensor Cysteines in Human Keap1 Modified by the Cancer Chemopreventive Agent Sulforaphane. Chem. Res. Toxicol. 2005, 12, 1917-1926.

13. Hong, F.; Sekhar, K. R.; Freeman, M. L.; Liebler, D. C. Specific Patterns of Electrophile Adduction Trigger Keap1 Ubiquitination and Nrf2 Activation. J. Biol. Chem. 2005, 36, 31768-31775.

14. Gerhauser, C. Broad Spectrum Anti-Infective Potential of Xanthohumol from Hop (Humulus Lupulus L.) in Comparison with Activities of Other Hop Constituents and Xanthohumol Metabolites. Mol. Nutr. Food Res. 2005, 9, 827-831.

15. Gerhauser, C. Beer Constituents as Potential Cancer Chemopreventive Agents. Eur. J. Cancer 2005, 13, 1941-1954. 
16. Stevens, J. F.; Page, J. E. Xanthohumol and Related Prenylflavonoids from Hops and Beer: To Your Good Health! Phytochemistry 2004, 10, 1317-1330.

17. Albini, A.; Dell'Eva, R.; Vene, R.; Ferrari, N.; Buhler, D. R.; Noonan, D. M.; Fassina, G. Mechanisms of the Antiangiogenic Activity by the Hop Flavonoid Xanthohumol: NF-kappaB and Akt as Targets. FASEB J. 2006, 3, 527-529.

18. Kim, D. C.; Choi, S. Y.; Kim, S. H.; Yun, B. S.; Yoo, I. D.; Reddy, N. R.; Yoon, H. S.; Kim, K. T. Isoliquiritigenin Selectively Inhibits $\mathrm{H}(2)$ Histamine Receptor Signaling. Mol. Pharmacol. 2006, 2, 493-500.

19. Jang, D. S.; Park, E. J.; Kang, Y. H.; Hawthorne, M. E.; Vigo, J. S.; Graham, J. G.; Cabieses, F.; Fong, H. H.; Mehta, R. G.; Pezzuto, J. M. Kinghorn, A. D. Potential Cancer Chemopreventive Flavonoids from the Stems of Tephrosia Toxicaria. J. Nat. Prod. 2003, 9, 1166-1170.

20. Baba, M.; Asano, R.; Takigami, I.; Takahashi, T.; Ohmura, M.; Okada, Y.; Sugimoto, H.; Arika, T.; Nishino, H.; Okuyama, T. Studies on Cancer Chemoprevention by Traditional Folk Medicines. XXV. Inhibitory Effect of Isoliquiritigenin on Azoxymethane-induced Murine Colon Aberrant Crypt Focus Formation and Carcinogenesis. Biol. Pharm. Bull. 2002, 2, 247-250.

21. Yamazaki, S.; Morita, T.; Endo, H.; Hamamoto, T.; Baba, M.; Joichi, Y.; Kaneko, S.; Okada, Y.; Okuyama, T.; Nishino, H.; Tokue, A. Isoliquiritigenin Suppresses Pulmonary Metastasis of Mouse Renal Cell Carcinoma. Cancer Lett. 2002, 1, 23-30.

22. Cuendet, M.; Oteham, C. P.; Moon, R. C.; Pezzuto, J. M. Quinone Reductase Induction as a Biomarker for Cancer Chemoprevention. J. Nat. Prod. 2006, 3, 460-463.

23. Iwasaki, Y.; Morita, A.; Iwasawa, T.; Kobata, K. Sekiwa, Y.; Morimitsu, Y.; Kubota, K.; Watanabe, T. A Nonpungent Component of Steamed Ginger-[10]-Shogaol-Increases Adrenaline Secretion via the Activation of TRPV1. Nutr. Neurosci. 2006, 3-4, 169-178.

24. Burton, A. Chemoprevention: Eat Ginger, Rub on Pomegranate. Lancet Oncol. 2003, 12, 715 .

25. Ihlaseh, S. M.; de Oliveira, M. L.; Teran, E.; de Camargo, J. L.; Barbisan, L. F. Chemopreventive Property of Dietary Ginger in Rat Urinary Bladder Chemical Carcinogenesis. World J. Urol. 2006, 5, 591-596.

26. Manju, V.; Nalini, N. Chemopreventive Efficacy of Ginger, a Naturally Occurring Anticarcinogen during the Initiation, Post-Initiation Stages of
1,2 Dimethylhydrazine-induced Colon Cancer. Clin. Chim. Acta 2005, 1-2, 60-67.

27. Park, E. J.; Pezzuto, J. M. Botanicals in Cancer Chemoprevention. Cancer Metastasis Rev. 2002, 3-4, 231-255.

28. Dietz, B. M.; Kang, Y. H.; Liu, G.; Eggler, A. L.; Yao, P.; Chadwick, L. R.; Pauli, G. F.; Farnsworth, N. R.; Mesecar, A. D.; van Breemen, R. B. Bolton, J. L. Xanthohumol Isolated from Humulus Lupulus Inhibits Menadione-induced DNA Damage through Induction of Quinone Reductase. Chem. Res. Toxicol. 2005, 8, 1296-1305.

29. Tao, Y. Characterization of Cyclooxygenase-2 Inhibitors as Anti-Inflammatory Agents from Ginger Dietary Supplements and In Vitro Metabolism Studies of Gingerol-Related Compounds, Ph.D. dissertation; University of Illinois at Chicago, 2007; p. 127

30. Eggler, A. L.; Liu, G.; Pezzuto, J. M.; van Breemen, R. B.; Mesecar, A. D Modifying Specific Cysteines of the Electrophile-Sensing Human Keap1 Protein Is Insufficient to Disrupt Binding to the Nrf2 Domain Neh2. Proc. Natl. Acad. Sci. U. S. A. 2005, 29, 10070-10075.

31. Rybak, J. N.; Scheurer, S. B.; Neri, D.; Elia, G. Purification of Biotinylated Proteins on Streptavidin Resin: A Protocol for Quantitative Elution. Proteomics 2004, 8, 2296-2299.

32. Talalay, P.; De Long, M. J.; Prochaska, H. J. Identification of a Common Chemical Signal Regulating the Induction of Enzymes That Protect Against Chemical Carcinogenesis. Proc. Natl. Acad. Sci. U. S. A. 1988, 21 8261-8265.

33. Xue, F.; Cooley, L. Kelch Encodes a Component of Intercellular Bridges in Drosophila Egg Chambers. Cell 1993, 5, 681-693.

34. Santos, R. L.; Hassan, M. I.; Sikandar, S.; Lee, K.; Ali, G.; Martin, P. E. Jr.; Wambangco, M. A.; Ahmad, W.; Leal, S. M. DFNB68, a Novel Autosomal Recessive Non-Syndromic Hearing Impairment Locus at Chromosomal Region 19p13.2. Hum. Genet. 2006, 1, 85-92.

35. Sakurai, T.; Kanayama, M.; Shibata, T.; Itoh, K.; Kobayashi, A. Yamamoto, M.; Uchida, K.; Ebselen, A. Seleno-Organic Antioxidant, as an Electrophile. Chem. Res. Toxicol. 2006, 9, 1196-1204.

36. Eggler, A. L.; Luo, Y.; van Breemen, R. B.; Mesecar, A. D. Identification of the Highly Reactive Cysteine 151 in the Chemopreventive AgentSensor Keap1 Protein is Method-Dependent. Chem. Res. Toxicol. 2007, 10 (E-pub ahead of print). 